\title{
A Novel Power Output Model for Photovoltaic Systems
}

\author{
Chiou-Jye Huang ${ }^{\mathrm{a} *}$, Mao-Ting Huang ${ }^{\mathrm{a}}$, and Chung-Cheng Chen ${ }^{\mathrm{b}}$ \\ ${ }^{a}$ Industrial Technology Research Institute, Hsinchu, Taiwan, R.O.C. \\ ${ }^{b}$ Department of Electrical Engineering, Hwa Hsia Institute of Technology, Taiwan, R.O.C.
}

\begin{abstract}
This study proposes an efficient approach for the model of photovoltaic systems power output by employing evolutionary programming algorithm schemes. The PV system power output is primarily influenced by the weather, the solar irradiance and the module temperature. The EP algorithm is employed for adjusting the optimal value of the fill factor to achieve more accurate PV system (PV array model and PV inverter model) power output. A model of the PV systems can be established and the power outputted. To validate the developed PV system model, some measured data from two PV systems are utilized to make comparison with the proposed EP algorithm schemes. The modeling of the PV system is performed and the performance of our presented power output approach proves the effectiveness of the proposed method. More accurate results can be obtained by adjusting the fill factor parameter of the built model.
\end{abstract}

Keywords: Evolutionary programming, fill factor, photovoltaic systems

\section{Introduction}

In the past decades, large-scale photovoltaic system has been worldwide in the developed countries. The advantages of employing the photovoltaic plant generate electricity including no production of pollutants during operation, silent, long lifetime and low maintenance. Besides, solar energy is abundant, free, clean and inexhaustible. Especially, the grid connected applications represent the fastest growth of the photovoltaic market. There is an important point that the PV output affects the power system stability. How way can more accurately predict the power output of the photovoltaic power plants, and industry and academia also focus on more forecasting effectively? Forecasting the power output of photovoltaic power plant research and applications can be classified into two categories: one is based on the intensity of solar irradiation prediction model. The solar irradiation model, being established according to the local weather history data, is the predicted value of the solar irradiation. However, it considers the model of the inverter. Finally, the PV system output can be predicted, such as [1]-[3], [6],[7], [10]-[12]. The model is dependent on the detailed meteorological data. While we want to require more accurate forecasting results, the model is more complex to be required the amount of the history of the weather data and more data types. It makes the prediction process be very cumbersome and not conducive to the realization of the actual situation.

The goal of another forecasting method is to predict the output of the PV power stations. The PV power stations and grid connected operation of photovoltaic panels, geographical location, environment and inverter systems have been identified by historical operating data for a reasonable statistical model predicting the PV system output power directly. It needs to eliminate a large number of the meteorological statistical process and twice complex modeling process or several times, so it can simplify

\footnotetext{
* Manuscript received April 27, 2012; revised August 20, 2012.

Corresponding author. Tel.: +886-3-5912210; fax:+886-3-5820218; E-mail address: chioujye@itri.org.tw.
} 
the prediction power output process. Under varying operating conditions, the objective of this paper is to pursue a model with acceptable precision estimating the actual performance of the PV system.

Evolutionary programming [2],[3],[5] is a parallel and global search technique that take the concepts from evolution theory and natural genetics. EP search algorithms are quite robust and good at finding an acceptable solution in a large search space. No need for prior knowledge of the dynamics is assumed and no derivative or environment information is necessary. The only concern for EP is the quality of the solution produced by each parameter set.

In this paper, we develop a method to power output of PV system based on EP algorithm. It is organized as the follows. In section II, we address the PV model dynamics, where a six parameters model is built up. Section III presents the designing process of the EP algorithm. In section IV, to validate the developed model, measured data from two PV systems in Taiwan are utilized to make comparison with the proposed EP schemes. Conclusions with discussions are given in section V.

\section{Photovoltaic System Modeling}

Some important electrical characteristics of a PV module are the short circuit current, the open circuit voltage, the fill factor and the maximum power output. Their functions depend on the solar irradiance intensity and the PV-module temperature. The six electrical characteristics are modelled as follows.

\subsection{Short circuit current}

The short circuit current $I_{s c 1}$ depends on the PV module temperature and solar irradiance. The equation of $I_{s c 1}$ for the PV module is written as follows [4], [5], [8], [11]:

$$
I_{s c 1}=I_{s c 0} \times\left[1+\alpha\left(T_{1}-T_{0}\right)\right] \times\left(G_{1} / G_{0}\right)
$$

where $I_{s c 0}$ is the short circuit current of the PV module in the standard solar irradiance $G_{0}$. While the environment changes with the solar irradiance $G_{1}$, the short circuit current is $I_{s c 1}$. $\alpha$ is the PV module electrical specification and it's unit is $\mathrm{A} /{ }^{\circ} \mathrm{C}$.

\subsection{Open circuit voltage}

The main relationship of the open circuit voltage for module temperature is to follow the PV module electrical specification $\beta$, and the open circuit can be expressed as follow [4], [8], [11]:

$$
V_{o c 1}=V_{o c 0} \times\left[1+\beta\left(T_{1}-T_{0}\right)\right]
$$

where the open circuit voltage at $V_{o c 1}$ is to be expressed by the open circuit voltage under STC conditions $V_{\text {oc0 }}$.

\subsection{Fill factor}

The fill factor is defined as $V_{m p} I_{m p} / V_{o c 1} I_{s c 1}$, where $V_{m p}$ and $I_{m p}$ represent the voltage and current at the maximum power point, respectively. It is defined as follow.

$$
F F=V_{m p} I_{m p} / V_{o c 1} I_{s c 1} .
$$

\subsection{Maximum power output}

The maximum power point on the I-V curve is to be produced by the cell. Although the voltage at any point on the graph can still be calculated by using $P=I \times V$. Maximum power point occurs on the "knee" of the $I-V$ curve. Since the fill factor determines the power output of the cell, the maximum power output relationship is given by: 


$$
P_{\max }=F F \times V_{o c 1} \times I_{s c 1} .
$$

\subsection{Photovoltaic array}

The PV system is composed of a combination of photovoltaic modules. It is connected in series and in parallel PV modules and extended the voltage and current, respectively. If a matrix of the $M_{s} \times M_{p} \mathrm{PV}$ modules is considered, the voltage and current scaling are given as follows:

$$
\begin{gathered}
I_{A}=M_{p} \times I_{M} \\
V_{A}=M_{s} \times V_{M}
\end{gathered}
$$

where $I_{A}$ and $V_{A}$ are the PV array current and voltage, respectively; $I_{M}$ and $V_{M}$ are the PV module current and voltage. From equations (3)-(6), the maximum power output of the PV array can be expressed by

$$
P_{A}=F F_{A} \times V_{A} \times I_{A}=M_{p} \times M_{S} \times P_{M},
$$

where $P_{A}, P_{M}$ are the PV array and PV module power output, respectively.

\subsection{Photovoltaic system}

The PV array power generates the DC component output, so the PV inverter performs the conversion of the variable DC output of the PV modules into a utility frequency AC current that can be fed into the commercial electrical grid or used by a local, off-grid electrical network. Therefore, the conversion efficiency of the PV inverter will affect the AC component of output power. The maximum power output of the PV system can be expressed by

$$
P_{S}=\eta_{I N V} \times P_{A} .
$$

where $\eta_{I N V}$ is PV inverter efficiency.

The structure of EP based on the output power of photovoltaic system is shown in the Fig. 2-1.

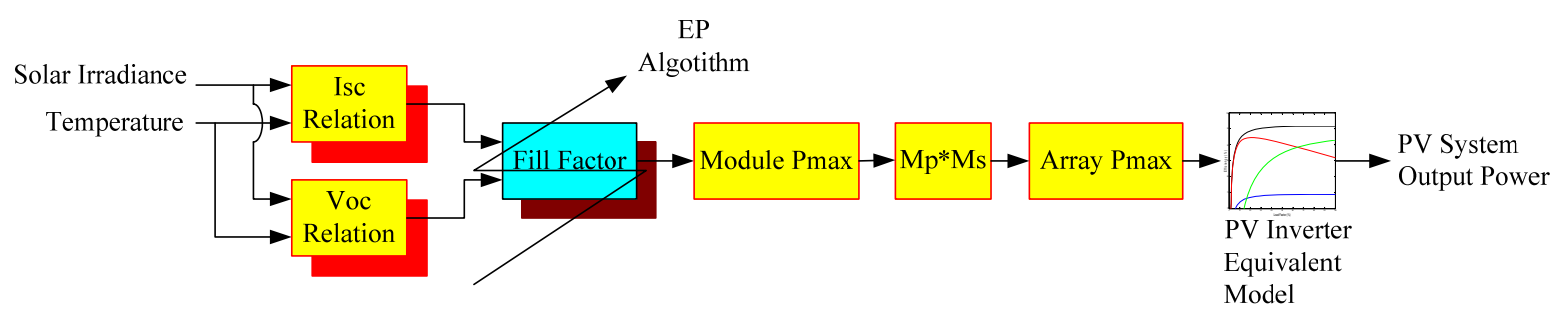

Fig. 2-1. Structure of EP based on the output power of photovoltaic system model.

\section{Evolutionary Programming Algorithm}

Evolutionary programming algorithm, which was first proposed by Fogel et al. [7], is a class of biologically inspired artificial intelligence search technique that can be applied to a variety of optimization tasks and problems. Basically a population of possible solutions is maintained, and this population evolves over a number of generations with "survival of the fittest" enabling the fitter members to breed with each other and replace less fitting members. The population strategy enables EP to search the near optimal solutions from various parts and directions within a search space simultaneously. Therefore, it can avoid converging to the local minimum or maximum points. The flowchart of EP procedure is shown in Fig. 3-1. 


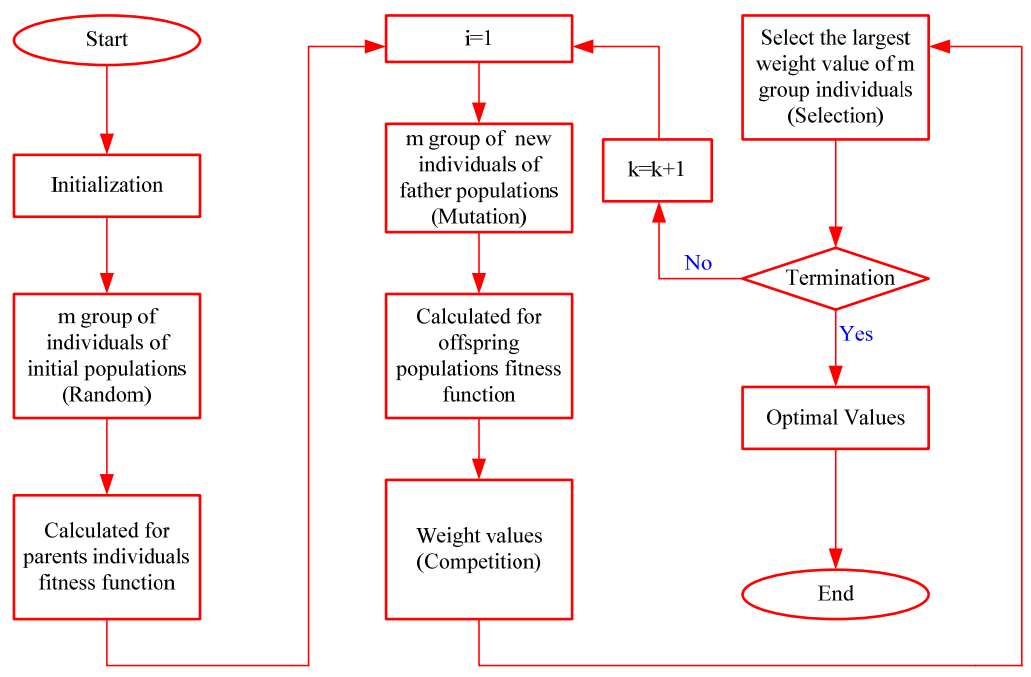

Fig. 3-1. The flowchart of EP algorithm.

The proposed EP procedures are implemented as follows:

Step 1. In the procedure of EP coding, we have adopted the binary type to code the population. We use a vector as a chromosome to present real values of the variables. The length of the vector depends on the precision of requirement. The mapping from a binary string $p=\left[\begin{array}{llll}a_{0} & a_{1} & \cdots & a_{N-1}\end{array}\right]$ of $\mathrm{N}$ bits into a real number is as

$$
F F=F F_{\text {min }}+\frac{\sum_{i=0}^{N-1} a_{i} 2^{i}}{2^{N}-1}\left(F F_{\text {max }}-F F_{\text {min }}\right)
$$

where $F F \in\left\{F F_{\text {min }} \quad F F_{\text {max }}\right\}$ is the real value to be encoded, $F F_{\text {min }}$ and $F F_{\text {max }}$ are the lower and upper bounds of the variables, respectively.

Step 2. From quasi-random sequence (QRS) [3], we can generate chromosomes as the initial population $\left[\begin{array}{llll}a_{0} & a_{1} & \cdots & a_{N-1}\end{array}\right]$ of each parameter of EP-based algorithm. Each chromosome is a binary vector of $\mathrm{N}$ bits. Then we can search for $F F$, which will create a $1 \mathrm{~N}$-dimensioal vector of representing the parameters. The fitness function of the system is defined as ISE,

$$
J_{i}\left(p_{i}\right)=\int_{0}^{t_{f}}\left[w_{1}\left(P_{\text {error }}\right)^{2}\right] d t .
$$

where $P_{\text {error }} \equiv P_{\text {measured }}-P_{\text {forecast }}$.

Step 3. Mutate initial population $p_{i}, i=1,2, \cdots, n$, double the population size from $\mathrm{n}$ to $2 n$, and create $p_{i+n}$ as follows:

$$
p_{i+n}=\left\{\begin{array}{l}
\text { two-bits-complement of } p_{i}, \text { if } J\left(p_{i}\right) \geq J_{\text {mean }}, \\
\text { one-bit-complament of } p_{i}, \text { if } J\left(p_{i}\right)<J_{\text {mean }}
\end{array}\right.
$$

where $J_{\text {mean }}$ is the mean value of ISE performance of all $J_{i}\left(p_{i}\right)$ that is from Step 2, one bit complement of $p_{i}$ represents that we select one bit of $p_{i}$ randomly and take the complement of that bit, and two bits complement of $p_{i}$ is defined in a similar way.

Step 4. Calculate the fitness score $J_{i}\left(p_{i+n}\right)$ for each $p_{i+n}, i=1,2, \cdots, n$, and rank the fitness scores of $J_{i+n}\left(p_{i+n}\right)$, for the next generation in selecting $\mathrm{n}$ individuals, where their ISE performance belongs to the $\mathrm{n}$ smallest ones.

Step 5. Repeat Step 3 until the best chromosome is not replaced in next ten generations. 


\section{Validation Results and Discussion}

\subsection{System introduction}

The model of the PV power output is verified with measured data for the two photovoltaic systems. We decide the location of the Tong Feng Junior High School (TFJHS), Bai He Library (BHL), which have been build since the year 2007 and 2009, respectively.

The PV system consists of $18 \mathrm{PV}$ modules each of $175 \mathrm{~W}$ and a SMA/SB3800 inverter in the TFJHS. The system described in Table 4-1 has more information. The BHL PV system is composed of 42 PV modules each of 120W. Table 4-2 has more detailed information in the BHL. Fig. 4-1 and Fig. 4-2 show the PV system installed in the TFJHS and BHL, respectively.

Table 4-1. Information of the PV System for TFJHS

\begin{tabular}{lllll}
\hline \multirow{2}{*}{ System } & Rated Capacity & Angle & Azimuth & Structure \\
& $3.15 \mathrm{kWp}$ & 23.5 degrees & South & 9 series 2 parallel \\
\hline \multirow{2}{*}{ Module } & Company/No. & P maximum & Voc & Isc \\
& Sharp/NT-R5E3E & $175 \mathrm{~W}$ & $44.4 \mathrm{~V}$ & $5.40 \mathrm{~A}$ \\
\hline \multirow{2}{*}{ Inverter } & Company/No. & Input Voltage & Output Power & Output Voltage \\
& SMA/SB3800 & $200 \sim 400 \mathrm{~V}$ & $3.8 \mathrm{~kW}$ & $1 \varphi 2 \mathrm{~W}$ AC 220V \\
\hline
\end{tabular}

Table 4-2. Information of the PV system for BHL

\begin{tabular}{lllll}
\multirow{2}{*}{ System } & Rated Capacity & Angle & Azimuth & Structure \\
& $5.04 \mathrm{kWp}$ & 6 degrees & South & 21 series 2 parallel \\
Module & Company/No. & P maximum & Voc & Isc \\
Kyocera/KC120 & $120 \mathrm{~W}$ & $21.5 \mathrm{~V}$ & $7.45 \mathrm{~A}$ \\
Inverter & Company/No. & Input Voltage & Output Power & Output Voltage \\
& SMA/SMC6000A & $246 \sim 480 \mathrm{~V}$ & $6 \mathrm{~kW}$ & $1 \varphi 2 \mathrm{~W}$ AC 220V \\
\hline
\end{tabular}

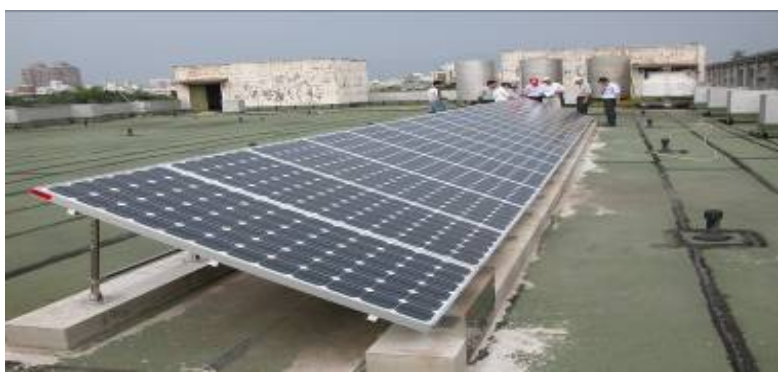

Fig. 4-1. PV system installed in the TFJHS.

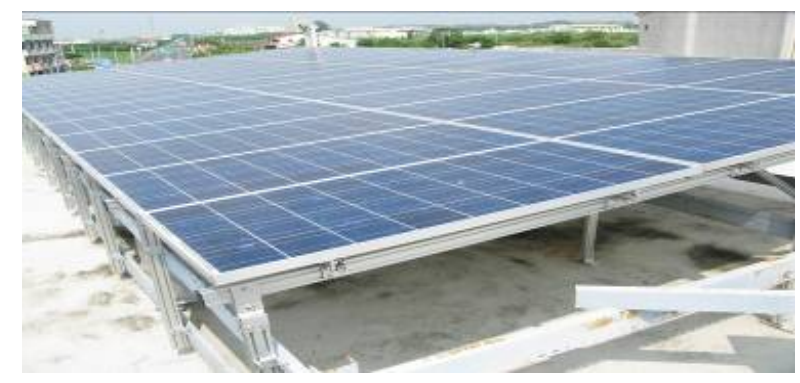

Fig. 4-2. PV system installed in the BHL.

\subsection{Analysis of photovoltaic inverter performance}

The PV inverter is critical component in solar energy systems. The PV inverter can convert the direct current generated by PV system into alternating current. The energy load factor $\left(e_{L R}\right)$ is PV array output power $\left(e_{D C}\right) / \mathrm{PV}$ inverter max DC input power $\left(e_{I N V_{-} M}\right)$. So that, the energy load factor can be expressed by

$e_{L R}=\frac{e_{D C}}{e_{I N V_{-} M}}$

Fig. 4-3 shows the efficiency characteristics of the PV inverter installed in TFJHS.

$\eta_{I N V}=\frac{e_{A C}}{e_{D C}}$

where $e_{D C}$ is $\mathrm{PV}$ array DC output power and $e_{A C}$ is the PV system AC output power and $\eta_{I N V}$ is the PV inverter efficiency. 


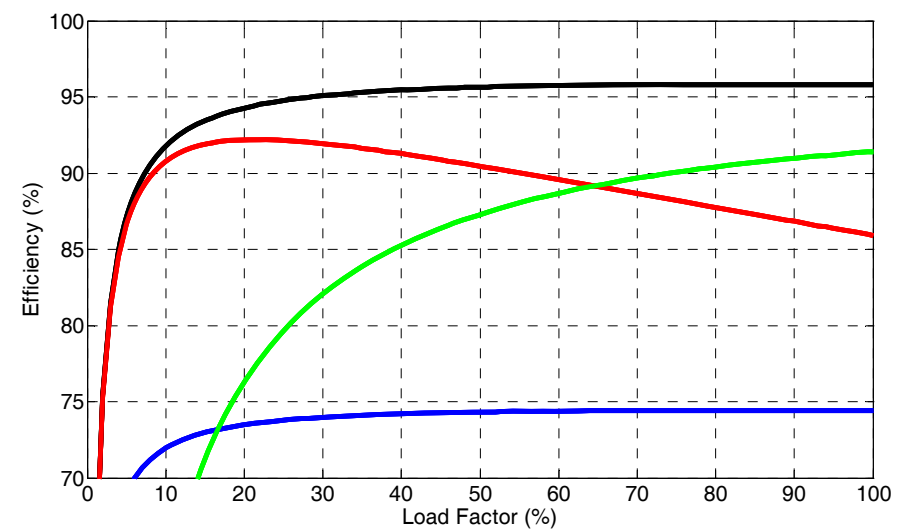

Fig. 4-3. Characteristic inverter efficiency curve by changing parameter of equation (13).

Table 4-3. The coefficients of PV inverter efficiency characteristics

\begin{tabular}{ccc}
\hline Systems coefficients & TFJHS & BHL \\
\hline A & 0.0650 & 0.0254 \\
B & 1.0250 & 1.0298 \\
C & 0.0070 & 0.0017 \\
\hline
\end{tabular}

To evaluate and analyze the performance of the PV inverter, as shown in Fig. 4-3, relationship between DC input power load factor and efficiency of the PV inverter is approximated by the following nonlinear regression [8],[9]:

$\eta_{I N V}=\frac{e_{L R}}{A \cdot e_{L R}^{2}+B \cdot e_{L R}+C}$

where $\eta_{I N V}$ is the estimated PV inverter efficiency, $e_{L R}$ the measured DC input power load factor and A, $\mathrm{B}, \mathrm{C}$, are the regression coefficients of the nonlinear equation. The regression coefficient " $\mathrm{A}$ " dominates the horizontal part of the PV inverter efficiency curve of the slope. The regression coefficient " $\mathrm{B}$ " dominates the horizontal part of the PV inverter efficiency curve of the vertical height. The regression coefficient " $\mathrm{C}$ " dominates the PV inverter efficiency curve of the knee point. Fig. 4-3 is the characteristic inverter efficiency curve by changing parameter of equation (13). We can observe the curve to shift from basic black to red by changing in parameter "A". The curve shifts from basic black to blue by changing in parameter " $B$ ". In the same way, the curve shifts from basic black to green by changing in parameter "C". Finally, the Table 4-3 shows the PV inverter model parameters for TFJHS and BHL experimental sites. Fig. 4-4 is TFJHS PV inverter efficiency of the actual measured value and the PV inverter modelling curve, where the yellow points are measured values, the pink line is modelling curve. It is obvious to see that we can get good modelling results.

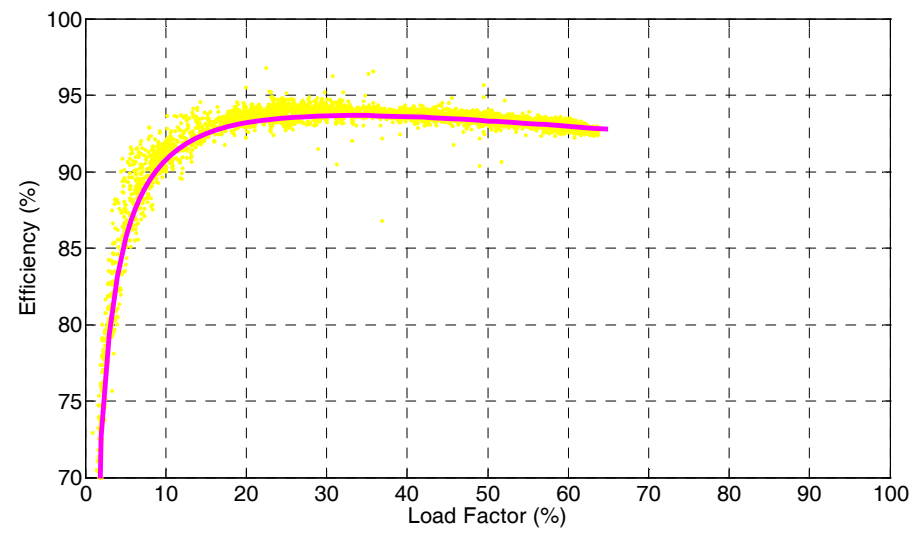

Fig. 4-4. TFJHS PV inverter efficiency of the measured value and the PV inverter model curve. 


\subsection{Discussion}

The verification results consider two kinds of the weather conditions, the sunny and cloudy. We choose two experiment stations to be TFJHS and BHL. Fig. 4-5 to Fig. 4-6 are based on the TFJHS experiment station. Fig. 4-7 to Fig. 4-8 are based on the BHL experiment station. The comparison between measured (blue line) and simulated by EP algorithm (red line) power output is shown in Fig. 4-5 and Fig. 4-7. The scatter plots of the measured and simulated power output are compared with Fig. 4-6 and Fig. 4-8.
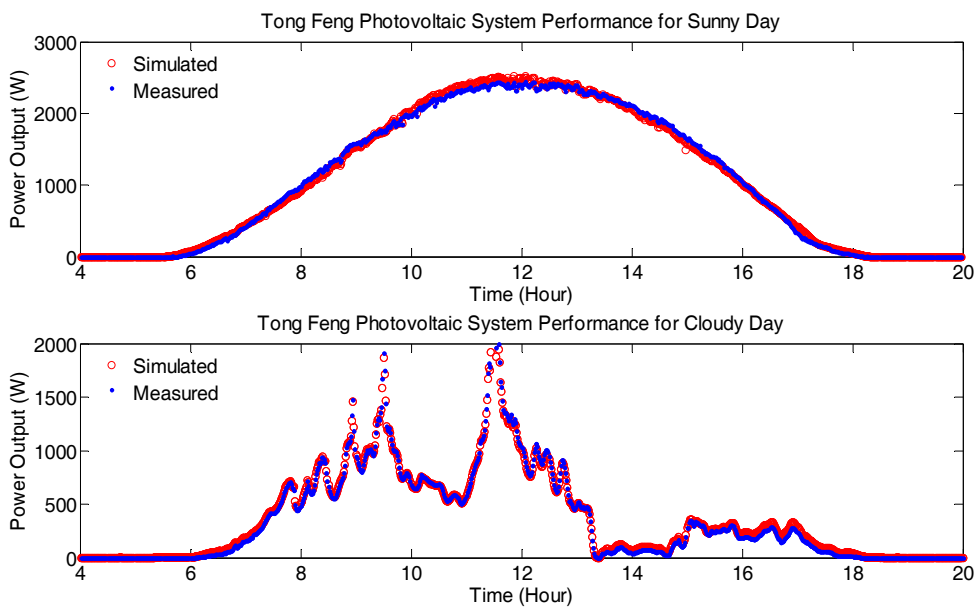

Fig. 4-5. Comparison between the measured and simulated power output at TFJHS.
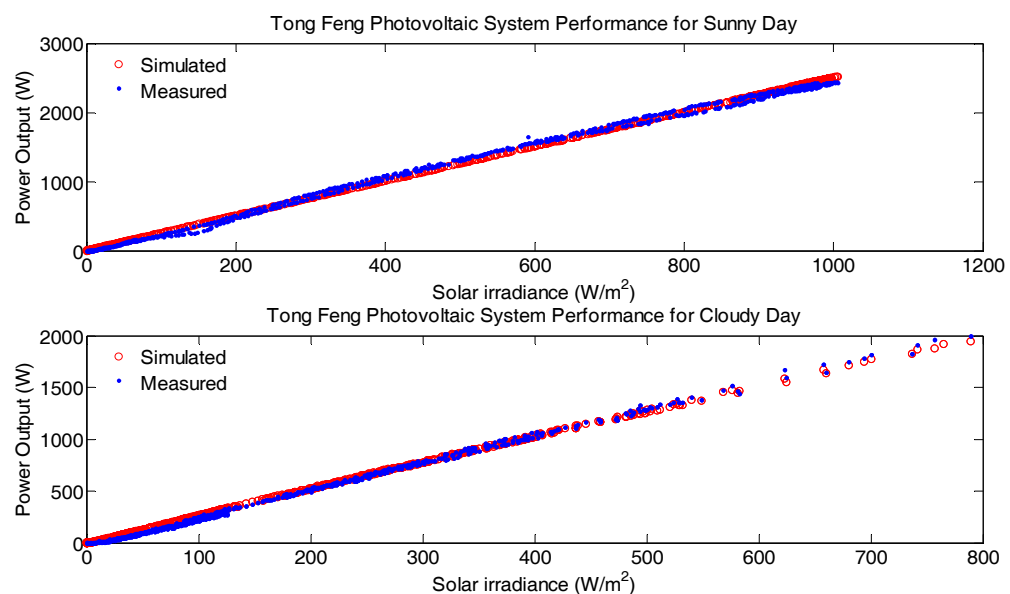

Fig. 4-6. Correlation between the measured and simulated power output at TFJHS.
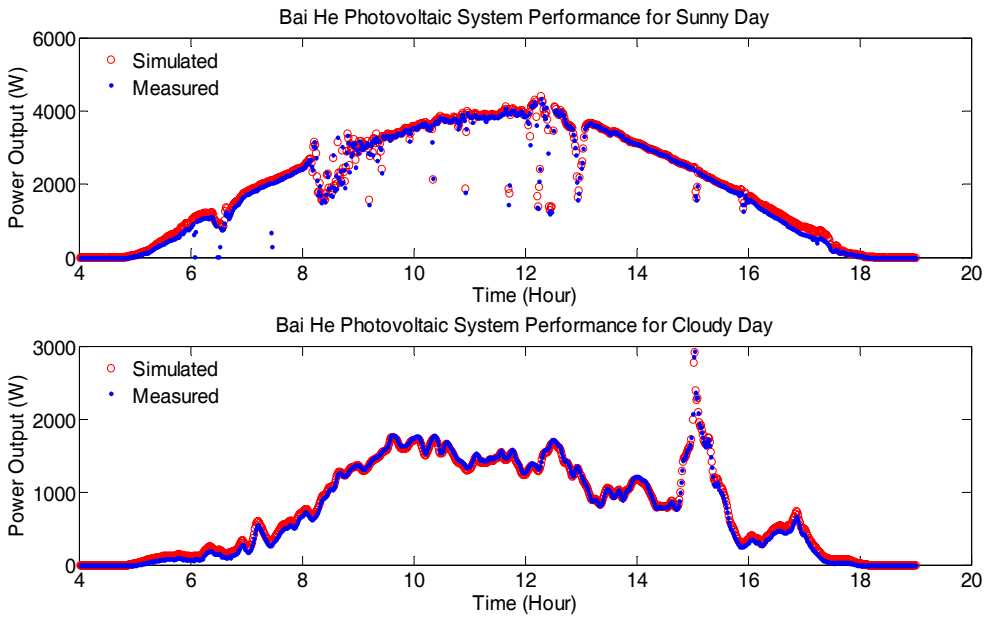

Fig. 4-7. Comparison between the measured and simulated power output at BHL. 

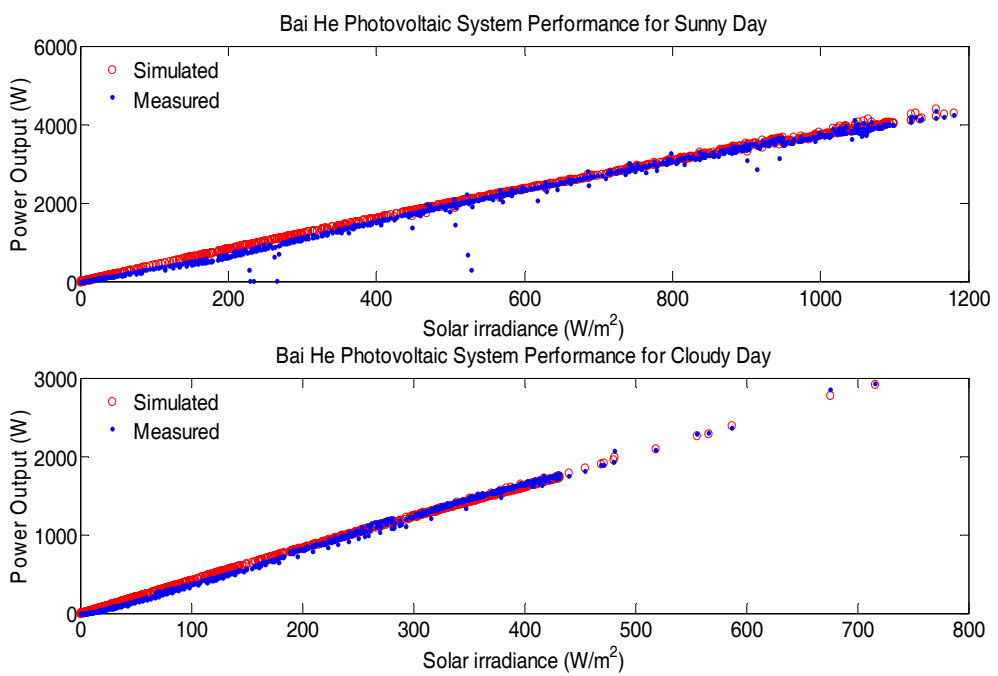

Fig. 4-8. Correlation between the measured and simulated power output at BHL.

The simulated power output is followed the trend of the measured data quite well in the Fig 4-5, and the simulated power output is followed the trend of the measured data quite well in the Fig 4-6. Fig. 4-6 illustrates the correlation between simulated and measured power output in TFJHS experimental station. In Fig. 4-6, the results for the sunny and cloudy conditions show the low solar irradiance the measured and simulated power output can be found ( $200 \mathrm{~W} / \mathrm{m}^{2}$ above) relatively high accuracy. Fig. 4-6 shows the measured and simulated values employing the coefficient of determination and R-square are $99.79 \%$ and $99.59 \%$ in sunny and cloudy conditions respectively.

The simulated power output of BHL experimental station is followed the trend of the measured data quite well in the Fig 4-8, and the simulated power output is followed the trend of the measured data quite well in the Fig 4-8. Fig. 4-8 illustrates the correlation between simulated and measured power output in BHL experimental station. In Fig. 4-8, the results for the sunny and cloudy conditions show the low solar irradiance the measured and simulated power output can be found $\left(600 \mathrm{~W} / \mathrm{m}^{2}\right.$ and $300 \mathrm{~W} / \mathrm{m}^{2}$ above for the sunny and cloudy conditions, respectively) relatively high accuracy. Fig. 4-8 shows the measured and simulated values employing the coefficient of determination and R-square are $99.08 \%$ and $99.62 \%$ in sunny and cloudy conditions respectively.

The above result exploits a fact that our EP based PV power output forecasting techniques have quite well accuracy. More detailed data shown in Table 4-4 for experimental results.

Table 4-4. The coefficient of determination for two experimental stations

\begin{tabular}{ccc}
\hline \hline$R^{2}$ & TFJHS & BHL \\
\hline Sunny & $99.79 \%$ & $99.08 \%$ \\
Cloudy & $99.59 \%$ & $99.62 \%$ \\
\hline \hline
\end{tabular}

\section{Conclusions}

The power output performance of the PV system is primarily influenced by the weather, the solar irradiance and the module temperature. This paper presents the photovoltaic system power output model based on EP algorithm method. More accurate power output results can be obtained by adjusting the FF parameter of the PV system model building. Numerical simulation results show that our proposed approach is effective and feasible in time under the same accuracy. The statistics of time increasing the amount can improve the modeling accuracy. Our EP based power output prediction results of photovoltaic system have clearly demonstrated that the R-square performance is very well. Finally, high coefficients of the determination R-square for two kinds weather conditions (sunny and cloudy day) demonstrate the good performance of the PV system power output model. 


\section{Acknowledgements}

This work is supported by the Bureau of Energy, Ministry of Economic Affairs, Taiwan, R.O.C., under contract No. 101-D0109.

\section{References}

[1] Deshmukh MK, Deshmukh SS. Modeling of hybrid renewable energy systems. Renewable and Sustainable Energy Reviews, 2008; 12(1):235-249.

[2] Eiben AE, Hinterding R, Michalewicz Z. Parameter control in evolutionary algorithms. IEEE Trans. Evolutionary Computation, 1999; 3(2):124-141.

[3] Fogel LJ, Owens AJ, Walsh MJ. Artificial Intelligence through Simulated Evolution. Chichester: Wiley, 1966.

[4] Hove T. A method for predicting long-term average performance of photovoltaic systems. Renewable Energy, 2000; 21(2):207-229.

[5] Kim JH, and Myung H. Evolutionary programming techniques for constrained optimization problems. IEEE Trans. Evolutionary Computation, 1997; 1(2):129-140.

[6] Mellit A, Pavan AM. Performance prediction of $20 \mathrm{kWp}$ grid-connected photovoltaic plant at Trieste (Italy) using artificial neural network. Energy Conversion and Management, 2010; 51(5): 2431-2441.

[7] Mellit A, Pavan AM. A 24-h forecast of solar irradiance using artificial neural network: application for performance prediction of a grid-connected PV plant at Trieste, Italy. Solar Energy, 2010; 84(5):807-821.

[8] Mondol JD, Yohanis YG, Smyth M, Norton B. Long-term validated simulation of a building integrated photovoltaic system. Solar Energy, 2005; 78(2):163-176.

[9] Oozeki T, Izawa T, Otani K, Tsuzuku K, Koike H, Kurokawa K. An evaluation method for PV systems by using limited data item. IEEJ Trans. on Power and Energy, 2006; 125(12):1299-1307.

[10] Picault D, Raison B, Bacha S, de la Casa J, Aguilera J. Forecasting photovoltaic array power production subject to mismatch losses. Solar Energy, 2010; 84(7):1301-1309.

[11] Tina G, Gagliano S, Raiti S. Hybrid solar wind power system probabilistic modeling for long-term performance assessment. Solar Energy, 2006; 80(5): 578-588.

[12] Zhou W, Yang H, Fang Z. A novel model for photovoltaic array performance prediction. Applied Energy, 2007; 84(12):11871198. 